\title{
Thermally stimulated conductivity in InGaAs/GaAs quantum wire heterostructures
}

\author{
S.A. Iliash ${ }^{1}$, S.V. Kondratenko ${ }^{1}$, A.S. Yakovliev ${ }^{1}$, Vas.P. Kunets ${ }^{2}$, Yu.I. Mazur ${ }^{2}$, and G.J. Salamo ${ }^{2}$ \\ ${ }^{1}$ Taras Shevchenko National University of Kyiv, \\ 64/13, Volodymyrs'ka str., 01601 Kyiv, Ukraine \\ E-mail: iliashsviatoslav@gmail.com \\ ${ }^{2}$ Institute for Nanoscience and Engineering, University of Arkansas, \\ Fayetteville, AR 72701, USA
}

\begin{abstract}
Thermally stimulated conductivity of the InGaAs-GaAs heterostructures with quantum wires was studied using different quantum energies of exciting illumination. The structures reveal long-term photoconductivity decay within the temperature range 100 to $200 \mathrm{~K}$, and effect of residual conductivity after turning-off the illumination. Analyzing the data of thermally stimulated conductivity, the following energies of electron traps were found: 90,140 , and $317 \mathrm{meV}$. The role of deep traps in recombination process as well as the photoconductivity mechanism was discussed.
\end{abstract}

Keywords: heterostructure, quantum wire, photoconductivity.

Manuscript received 24.11.15; revised version received 03.02.16; accepted for publication 16.03.16; published online 08.04.16.

\section{Introduction}

Nowadays the interest in studying the physical properties of heterostructures InGaAs-GaAs, specifically centers with deep and shallow trap levels, has increased significantly [4-7]. It is caused by its successful use to solve the scientific and technical problems for several years. InGaAs-GaAs heterostructure with quantum wires (QWR) are known for their unique photoelectrical properties that are perspective for implementation of new phototransistor technologies, infrared photodetectors and solar cells.

Like to other semiconductor materials, deep defect states in the band gap of InGaAs are formed by doping with a wide class of impurities [5]. These states may be significantly complicated due to formation of dopant pairs or their interaction with their own lattice defects. Optical and electronic properties are studied by photocurrent (PC) spectra and thermal activation effects measurement [3]. However, thermally stimulated conductivity (TSC) spectra of InGaAs-GaAs hetero- structures with QWR are not examined enough. According to [1-3], distribution of localized states in semiconductors is based on the multiple trapping model. It was shown that peaks in the spectra TSC were observed within the temperature range $100 \ldots 150 \mathrm{~K}$.

The main goal of this work is to obtain TSC spectra for heterostructures InGaAs-GaAs and to interprete them. To reach the goal, the classic method for obtaining TSC spectra and periodic light excitation method during the heating from 83 to $276 \mathrm{~K}$ were used in this paper.

\section{Experimental details}

All heterostructures were grown on GaAs (311)A semiinsulating substrates by using molecular beam epitaxy. After growing the $0.5-\mu \mathrm{m} \mathrm{GaAs}$ buffer layer at $580{ }^{\circ} \mathrm{C}$, the substrate temperature was reduced down to $520^{\circ} \mathrm{C}$ for deposition of the InGaAs and $n$-type GaAs barriers. Five periods of InGaAs/GaAs were deposited with 6, 8, 10 and 11 monolayers (MLs) of $\mathrm{In}_{0.38} \mathrm{Ga}_{0.62} \mathrm{As}$. Each GaAs barrier was $40-\mathrm{nm}$ thick consisting of $10-\mathrm{nm}$ 
undoped GaAs, then 20-nm uniformly doped GaAs:Si $\left(N_{d}=5 \cdot 10^{17} \mathrm{~cm}^{-3}\right)$, and then another 10-nm undoped GaAs. Finally, each sample was capped with a final InGaAs QWR layer of equal deposition to the buried QWRs for AFM study. Samples with 11 and 10 MLs of InGaAs deposition are shown to form high-quality QWRs [7], while structures with 6 and 8 MLs maintain quantum well-like nature.

In order to get results, firstly, we cooled the sample to the temperature of $83 \mathrm{~K}$, then linearly heated and periodically lighted samples (for $30 \mathrm{~s}$ ). Spectra of lateral PC for $11 \mathrm{ML}$ InGaAs-GaAs QWR heterostructure measured within the range $0.6 \ldots 2.0 \mathrm{eV}$ at the temperature $83 \mathrm{~K}$. The time dependence of $\mathrm{PC}$ rise and decay obtained during excitation by using light with energies $h v_{1}=1.35 \mathrm{eV}$ and $h v_{2}=1.65 \mathrm{eV}$ within the temperature range 83 to $296 \mathrm{~K}$.

\section{Results and discussion}

Fig. 1 shows the time dependence of photoconductivity damping and decay, measured at different temperatures for InGaAs-GaAs structure with light excitation $h v_{1}=$ $1.35 \mathrm{eV}$ and $h v_{2}=1.65 \mathrm{eV}$. This experiment was performed under the condition of a linear temperature increase at the speed close to $0.05 \mathrm{~K} / \mathrm{s}$, and therefore, a change in temperature during the relaxation process is negligible (see Fig. 2a, insert). The kinetics of PC decay was described by a stretched exponential dependence:

$I_{P C} \sim \exp \{-(t / \tau) \beta\}$,

where $\tau$ is the time constant, $\beta$ - ideality factor, which is equal to $\beta=0.7$ for the studied structures. Note that the law proved to an exponential PC increase, and the ratio of time decay constant to the time increase one was $\tau_{\text {dec }} / \tau_{\text {rise }}=2.52$. It means that in addition to recombination centers, long term electronic traps (shallow trap centers) have a significant impact on PC decay $[4,6]$.

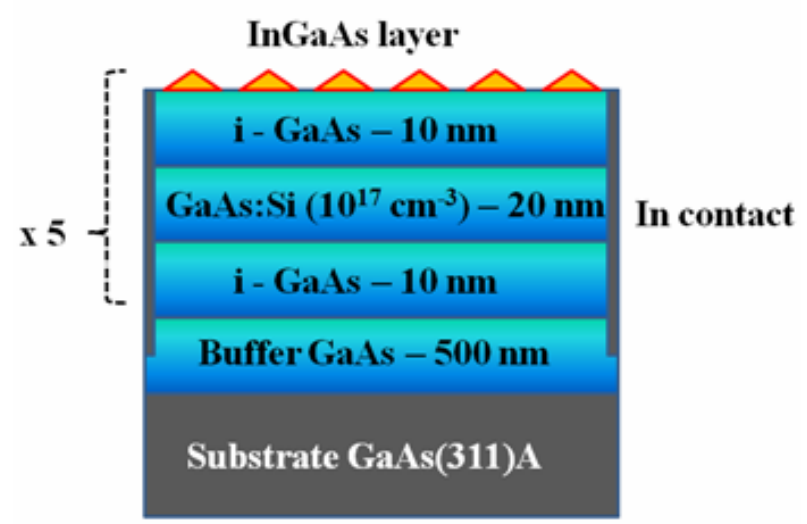

Fig. 1. Growth diagram of InGaAs-GaAs heterostructure.
Fig. 2a shows the extended relaxation (residual photoconductivity), when conductivity is not returned to its equilibrium value for a long $(\sim 30 \mathrm{~s})$ time, under excitation $h v_{1}=1.35 \mathrm{eV}$ at temperatures between 120 and $150 \mathrm{~K}$. We can distinguish two peaks of thermal conductivity in the dark background of conductivity (dashed curve), which is measured under similar experimental conditions, but without photoexcitation. Energy $h v_{1}$ and $h v_{2}$ were selected being based on the analysis of the spectral dependence of InGaAs-GaAs structure (Fig. 3), measured at $83 \mathrm{~K}$. Photocurrent spectroscopy reveals several electron transitions indicating the complicated density of states spectrum in our samples (Fig. 3). Besides electron transitions between ground states of conduction and valence bands of InGaAs QWR (arrow 1), the PC signal below band gap of GaAs is the result of photoexcited electrons in both the buffer layer (arrow 2) and defect states of GaAs (arrows 4 and 5) [4]. Band-to-band absorption in GaAs is observed above $1.43 \mathrm{eV}$ (arrow 3). The energy $h v_{1}=$ $1.35 \mathrm{eV}$ causes resonant excitation of InGaAs QWR as a result of band-to-band transitions, while $h v_{2}=1.65 \mathrm{eV}$ is also responsible for possible excitation of electron-hole pairs in GaAs. In addition, PC within the spectral range $0.7 \ldots 1.3 \mathrm{eV}$ is caused by transitions between GaAs deep states. These energies determined in [6] indicate the presence of deep levels in the system.

Fig. 4 shows the TSC curve for InGaAs-GaAs $11 \mathrm{ML}$ structure, measured by heating after excitation $h v_{1}=1.35 \mathrm{eV}$. We can select a wide peak of thermal conductivity, but in contrast to the kinetic method, we cannot distinguish the detail structure of the TSC spectrum.

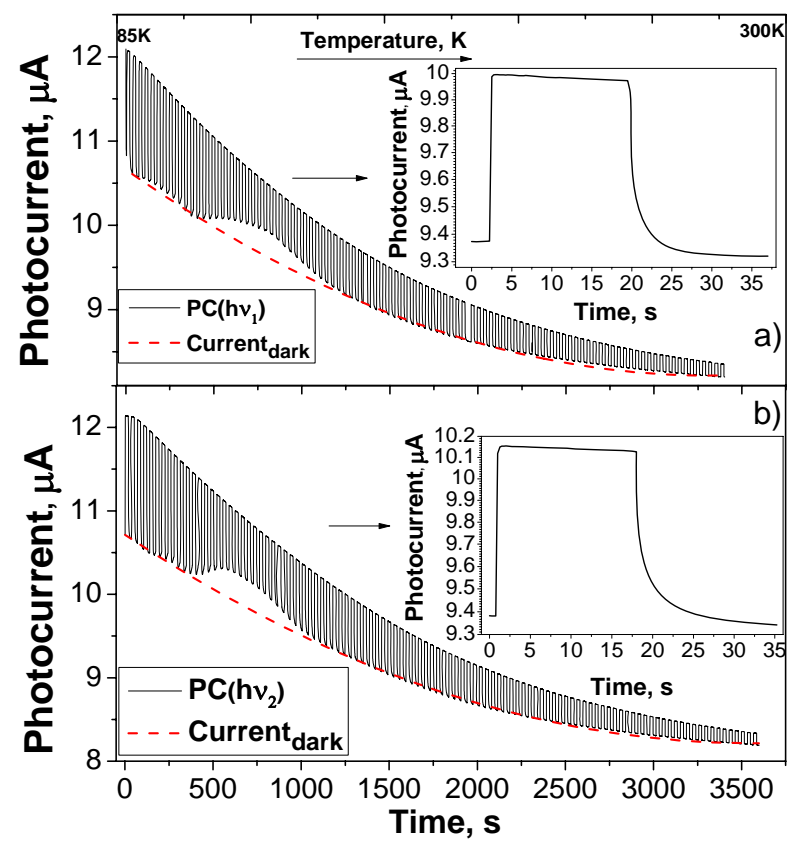

Fig. 2. Time dependences of PC relaxation under light excitation $h v_{1}(\mathrm{a})$ and $h v_{2}(\mathrm{~b})$ and various temperatures $(83 \ldots 276 \mathrm{~K})$. (The dashed line corresponds to the dark current.) 


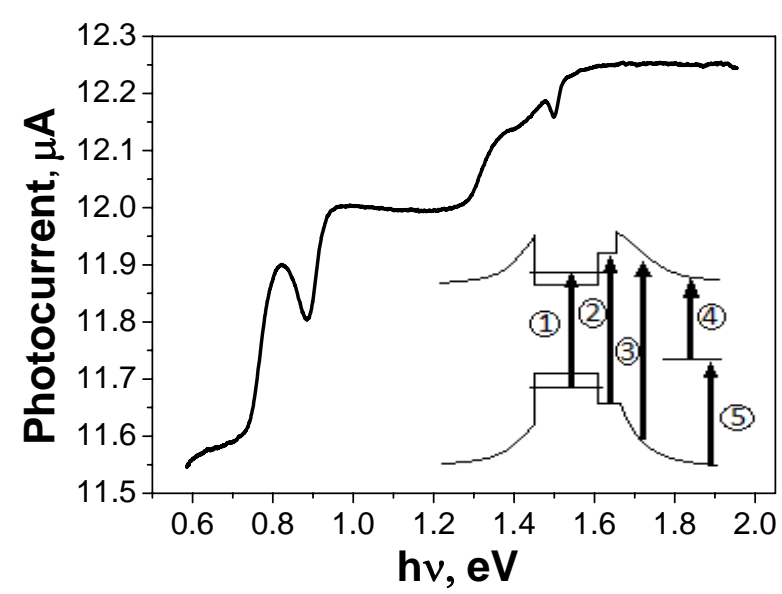

Fig. 3. Spectra of lateral PC for $11 \mathrm{ML}$ InGaAs-GaAs QWR heterostructure measured at $83 \mathrm{~K}$. The bias voltage $100 \mathrm{mV}$ was applied along QWR ( $[\overline{2} 33]$ direction).

TSC peak with a maximum at $160 \mathrm{~K}$ was observed within the temperature range from 130 up to $200 \mathrm{~K}$ (Fig. 4). Assuming that the recapture (capture other sticking centers) does not happen, we can estimate the depth of attachment center relative to the conduction band [5]:

$\varepsilon_{a}=23 k_{\mathrm{B}} T_{\max }$,

where $k_{\mathrm{B}}$ is the Boltzmann constant, $T_{\max }-$ temperature of the TSC peak. This formula is simplified and provides a relatively large error in assessing the activation energy. Using the equation (2), we can give rough estimate of the localization depth of charge carrier on a shallow center relatively to the center of the conduction band $\varepsilon_{a}=$ $317 \mathrm{meV}$.

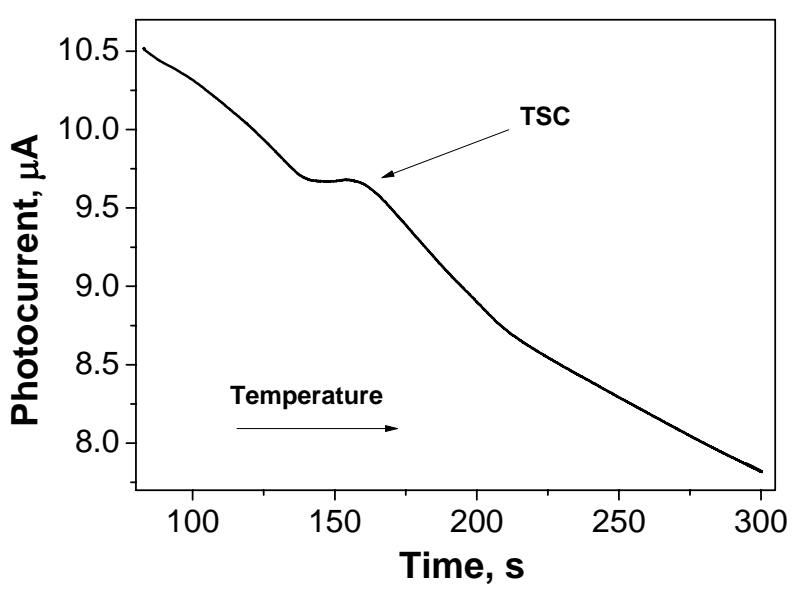

Fig. 4. Classical thermally stimulated conductivity for InGaAsGaAs 11 ML heterostructure.

Considering the shallow centers that are surrounded by QWR, it is possible that they determine the heterostructure conductivity in non-equal state. Due to the fact that the studied heterostructure has trapping center for electrons, it can be argued that the excited QWR have negatively charged environment.

Temperature dependence of PC decay shown in Figs. 5a, 5b for $h v_{1}=1.35 \mathrm{eV}$ and $5 \mathrm{c}$ for $h v_{2}=1.65 \mathrm{eV}$. We can see that in both cases two TSC peaks, but for $h v_{1}$ (Fig. 4c) the spectrum is more extended and shifted into the region of higher temperatures.

Fig. 5 shows that the activation energy for electrons at excitation $h v_{1}(1.35 \mathrm{eV})$ is equal to $\varepsilon_{a}=229 \mathrm{meV}$ for the first maximum and to $\varepsilon_{a}=337 \mathrm{meV}$ for the second one. The activation energy at excitation $h v_{2}(1.65 \mathrm{eV})$ is equal to $\varepsilon_{a}=218 \mathrm{meV}$ for the first maximum and to $\varepsilon_{a}=278 \mathrm{meV}$ for the second maximum.
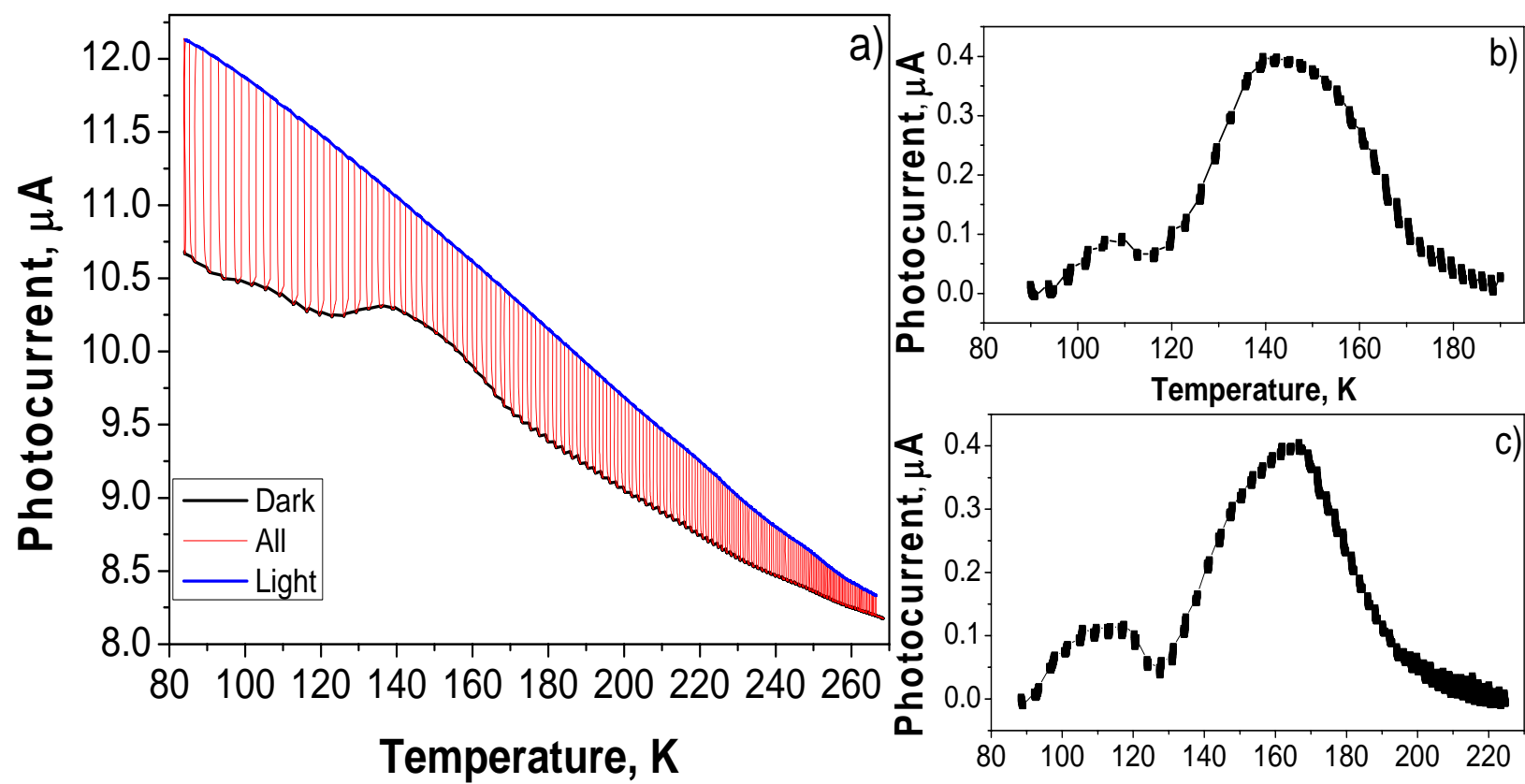

Fig. 5. (a) Dependence of the TSC spectra on heating (excitation $h v_{1}=1.35 \mathrm{eV}$ ). Thermally stimulated conductivity of the sample $11 \mathrm{ML}$ InGaAs-GaAs for various excitation intensities, (b) for $h v_{1}=1.35 \mathrm{eV}$, (c) for $h v_{2}=1.65 \mathrm{eV}$.

(C) 2016, V. Lashkaryov Institute of Semiconductor Physics, National Academy of Sciences of Ukraine 


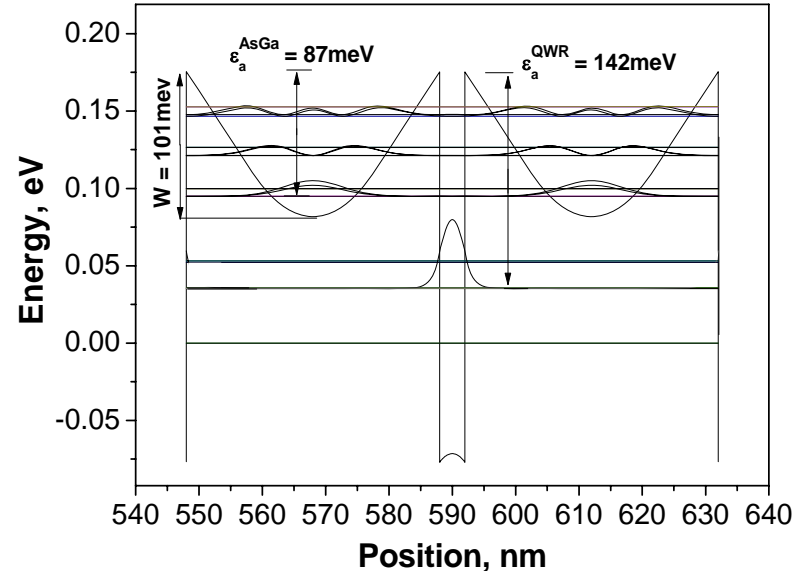

Fig. 6. Energy band diagram of the $11 \mathrm{ML} \operatorname{In}_{\mathrm{x}} \mathrm{Ga}_{1-\mathrm{x}} \mathrm{As}$ structure. Where $\mathrm{x}=0.38$, QWR height is $3.8 \mathrm{~nm}$.

When we use the formula (2) for assessing the activation energy, we obtain a sufficiently large error, because the formula is simplified and ignores the multiple trapping. The resulting activation energy was much higher than the values obtained from the analysis of TSC kinetic method. It means that at stationary filling TSC revealed deep electronic states.

Fig. 6 shows a diagram of the energy levels in the $11 \mathrm{ML} \operatorname{In}_{\mathrm{x}} \mathrm{Ga}_{1-\mathrm{x}} \mathrm{As}$ heterostructure, calculated using the software NextNano ${ }^{3}$ with the parameters $x=0.38$ and QWR height of $3.8 \mathrm{~nm}$. We revealed that the structure is characterized by potential variation caused by the intermediate layers of doped GaAs, which creates potential parabolic quantum wells $(W=101 \mathrm{meV})$. In addition, the system has quantum-sized states implied by the movement restriction of charge carriers in $\operatorname{In}_{x} \mathrm{Ga}_{1-\mathrm{x}} \mathrm{As}$ QWR. Thus, the system includes two subsystems of quantum-sized states due to the electron movement restriction in either GaAs buffer layer, or in $\operatorname{In}_{x} \mathrm{Ga}_{1-\mathrm{x}} \mathrm{As}$ QWR. According to band diagram calculations of the activation energy for GaAs and QWR, potential wells were equal to $\varepsilon_{a}=87 \mathrm{meV}$ and $\varepsilon_{a}=142 \mathrm{meV}$, respectively. Electrons are localized at a minimum of GaAs and can be spatially separated from the holes. These photoelectrons cause extended photocurrent relaxation, because for their recombination with holes in $\mathrm{In}_{\mathrm{x}} \mathrm{Ga}_{1-\mathrm{x}} \mathrm{As}$ it is necessary to overcome the potential barrier height $\varepsilon_{a}^{\mathrm{GaAs}}=87 \mathrm{meV}$ (see Fig. 6).

It was found that the temperature dependence of the time constant $\tau$ within the temperature range between 80 and $150 \mathrm{~K}$ agrees well with Arrhenius behavior of decay, when $\ln \tau(T) \sim \varepsilon_{a} / k T$. After analyzing the dependence of $\tau$ on temperature for $h v_{1}=1.35 \mathrm{eV}$ and $h v_{2}=1.65 \mathrm{eV}$, we obtained the activation energies $\varepsilon_{a}=146 \mathrm{meV}$ and $\varepsilon_{a}=92 \mathrm{meV}$, respectively. With account of the importance of energy activation for potential wells in GaAs and QWR, obtained from the band energy diagram, we conclude that electrons mainly fill the quantum states of QWR under excitation $h v_{1}$, and electrons fill GaAs potential well under excitation $h v_{2}$.
Consequently, we get the photocurrent relaxation processes involving different energy states (recombination centers) when light excitation with different energies $(1.35,1.65 \mathrm{eV})$ is used.

\section{Conclusions}

Thermally stimulated conductivity of the InGaAs-GaAs heterostructures with quantum wires was studied using different quantum energies of exciting illumination. The spectrum of electronic states that determines recombination in InGaAs-GaAs heterostructures was obtained at different temperatures, using the TSC method and kinetic one with periodic illumination of the sample. The structures reveal long-term photoconductivity decay within the temperature range $100 \ldots 200 \mathrm{~K}$ and effect of residual conductivity after turning-off the illumination. Analyzing the data of thermally stimulated conductivity, the following energies of electron traps were found: 90 , 140 , and $317 \mathrm{meV}$. The obtained activation energy for potential wells of the band structure and studied temperature dependence of the time constant $\tau$ show that light excitation with a selective energy for $\operatorname{In}_{x} \mathrm{Ga}_{1-\mathrm{x}} \mathrm{As}$ QWR leads to increase in PC caused by electrons concentrated in QWR.

\section{References}

1. H. Fritzsche, N. Ibaraki, Thermostimulated conductivity in amorphous semiconductors // Phil. Mag., Part B, 52(3), p. 299-311(1985).

2. S.C. Agarwal, H. Fritzsche // Phys. Rev. B, 10, p. 4351 (1974).

3. Gu. Benyuan, Zhengyi Xu, and Bizhen Dong, A theoretical interpretation of thermostimulated conductivity in amorphous semiconductors // J. Non-Crystalline Solids, 97, p. 479-482 (1987).

4. S.V. Kondratenko, O.V. Vakulenko, Vas.P. Kunets, Y.I. Mazur, V.G. Dorogan, M.E. Ware \& G.J. Salamo, Photoconductivity peculiarities in InGaAs quantum wire heterostructures: anisotropy and high photoresponsivity at room temperature // Semiconductor Sci. Technol. 27(10), 105024 (2012).

5. A.G. Milnes, Deep Impurities in Semiconductors. 1973.

6. O.V. Vakulenko, S.L. Golovins'kij, S.V. Kondratenko, Y. Mazur, Z.M. Vang \& G.D. Salamo, Effect of interface defect states on photoelectric properties of $\operatorname{In}_{\mathrm{x}} \mathrm{Ga}_{1-\mathrm{x}} \mathrm{As} / \mathrm{GaAs}$ heterostructures with quantum dots // Ukrainskyi Fizych. Zhurnal, 56(9), p. 944-952 (2011).

7. Vas.P. Kunets, S. Prosandeev, Y.I. Mazur, M.E. Ware, M.D. Teodoro, V.G. Dorogan \& G.J. Salamo, Isotropic Hall effect and "freeze-in" of carriers in the InGaAs self-assembled quantum wires // J. Appl. Phys. 110(8), 083714 (2011). 